Louisiana State University

LSU Digital Commons

Faculty Publications

Department of Geology and Geophysics

$2-28-2018$

\title{
Identifying apparent local stable isotope equilibrium in a complex non-equilibrium system
}

\author{
Yuyang $\mathrm{He}$ \\ Louisiana State University \\ Xiaobin Cao \\ Louisiana State University \\ Jianwei Wang \\ Louisiana State University \\ Huiming Bao \\ Louisiana State University
}

Follow this and additional works at: https://digitalcommons.Isu.edu/geo_pubs

\section{Recommended Citation}

He, Y., Cao, X., Wang, J., \& Bao, H. (2018). Identifying apparent local stable isotope equilibrium in a complex non-equilibrium system. Rapid Communications in Mass Spectrometry, 32 (4), 306-310. https://doi.org/10.1002/rcm.8040

This Article is brought to you for free and open access by the Department of Geology and Geophysics at LSU Digital Commons. It has been accepted for inclusion in Faculty Publications by an authorized administrator of LSU Digital Commons. For more information, please contact ir@lsu.edu. 


\title{
Identifying apparent local stable isotope equilibrium in a complex non-equilibrium system
}

\author{
Yuyang He*, Xiaobin Cao, Jianwei Wang, Huiming Bao
}

Department of Geology and Geophysics, Louisiana State University, E235 Howe Russell Kniffen

Baton Rouge, LA 70803, USA

* Corresponding author Email: yhe16@lsu.edu (Yuyang He)

\begin{abstract}
RATIONALE: Although being out of equilibrium, biomolecules in organisms have the potential to approach isotope equilibrium locally because enzymatic reactions are intrinsically reversible. A rigorous approach that can describe isotope distribution among biomolecules and their apparent deviation from equilibrium state is lacking, however.
\end{abstract}

METHODS: Applying the concept of distance matrix in graph theory, we propose that apparent local isotope equilibrium among a subset of biomolecules can be assessed using an apparent fractionation difference $(|\Delta \alpha|)$ matrix, in which the differences between the observed isotope composition $\left(\delta^{\prime}\right)$ and the calculated equilibrium fractionation factor $(1000 \ln \beta)$ can be more rigorously evaluated than by using a previous approach for multiple biomolecules. We tested our $|\Delta \alpha|$ matrix approach by re-analyzing published data of different amino acids (AAs) in potato and in green alga.

RESULTS: Our reanalysis shows that biosynthesis pathways could be the reason for an apparently close-to-equilibrium relationship inside AA families in potato leaves. Different biosynthesis/degradation pathways in tubers may have led to the observed isotope distribution difference between potato leaves and tubers. The analysis of data from green algae does not support the conclusion that AAs are further from equilibrium in glucose-cultured green algae than the autotrophic ones.

This is the author manuscript accepted for publication and has undergone full peer review but has not been through the copyediting, typesetting, pagination and proofreading process, which may lead to differences between this version and the Version of Record. Please cite this article as doi: $10.1002 / \mathrm{rcm} .8040$

This article is protected by copyright. All rights reserved. 
CONCLUSIONS: Application of the $|\Delta \alpha|$ matrix can help us to locate potential reversible reactions or reaction networks in a complex system such as a metabolic system. The same approach can be broadly applied to all complex systems that have multiple components, e.g. geochemical or atmospheric systems of early Earth or other planets.

Keywords: Multi-component system, thermodynamic order, disequilibrium, graph theory, apparent $\alpha$ difference matrix

\section{Introduction}

Living systems are open systems that exchange energy and matter with the environment. Life is inherently out-of-equilibrium chemically in general, and stable isotopically in particular, among its many constituents. However, the reversibility of enzymatic reactions is well recognized ${ }^{[1-3]}$. This leads to the possibility that an organism can attain local isotope equilibrium among or within certain biomolecules for some elements such as carbon if a subset of biomolecules is involved in fully reversible reactions ${ }^{[4-7]}$. By inference, biomolecules involved in the least reversible reactions should have isotope composition away from a thermodynamic equilibrium state $^{[4-6]}$. Thus, the deviation of the stable isotope composition of a set of biomolecules from their expected thermodynamic equilibrium state may be used as a measure of the degree of expressed reversibility in enzymatic reactions.

It was ${ }^{[4]}$ observed that, among or within some biomolecules in organisms, good linear correlation exists between carbon isotope composition $\left(\delta^{13} \mathrm{C}\right.$ values) and the corresponding ${ }^{13} \beta$ value ${ }^{[4]}$. Here, ${ }^{13} \beta$ is the reduced partition function ratio (RPFR), which is the predicted equilibrium isotope fractionation factor between a carbon compound or a site-specific carbon and the atomic carbon (i.e. the calculated equilibrium ${ }^{13} \mathrm{R}_{\mathrm{A}} /{ }^{13} \mathrm{R}_{\text {atomic }}$ value at a given temperature, where ${ }^{13} \mathrm{R}=$ $\left.\left[{ }^{13} \mathrm{C}\right] /\left[{ }^{12} \mathrm{C}\right]\right)$. The concept of $\beta$ or RPFR was proposed independently by Urey ${ }^{[8]}$ and Bigeleisen and Mayer ${ }^{[9]}$. It is a theoretical corner-stone of stable isotope geochemistry, which facilitates a universal comparison of equilibrium stable isotope fractionation between any compounds of interest.

The ${ }^{13} \beta-\delta^{13} \mathrm{C}$ correlation is described as:

$\delta^{13} C-\delta^{13} C_{\text {ave }}=\chi\left({ }^{13} \beta-{ }^{13} \beta_{\text {ave }}\right) \times 10^{3}$

where ave denotes the arithmetic average of $\delta$ or $\beta$ values for all the components of interest, and $\chi$ is the regression coefficient, reflecting the degree of "thermodynamic order" with respect to a complete equilibrium state when $\chi=1^{[4]}$. Some of the observed strong positive ${ }^{13} \beta-\delta^{13} \mathrm{C}$ correlations led Galimov $^{[4]}$ to propose that thermodynamic order or a certain degree of isotope equilibrium is sometimes present among biomolecules, although as a whole a living system is not in thermodynamic equilibrium. He pointed out that the fundamental reason behind the apparent thermodynamic order is the reversibility of enzymatic reactions. 
The idea that reversibility of enzymatic reactions is commonly expressed in biological systems was disputed ${ }^{[10]}$. In adddition, the cited positive ${ }^{13} \beta-\delta^{13} \mathrm{C}$ correlation as a valid evidence for the existence of close-to-equilibrium isotope distribution has been debated, since ${ }^{13} \beta-\delta^{13} \mathrm{C}$ correlation is not commonly observed in living systems and even, if an apparent ${ }^{13} \beta-\delta^{13} \mathrm{C}$ correlation is observed, thermodynamic control is not the only possible explanation ${ }^{[11-12]}$. For instance, a $\delta^{13} \mathrm{C}$ distribution of a set of molecules produced by non-equilibrium biochemical process (e.g. kinetic isotope effect) could also correlate with their corresponding ${ }^{13} \beta$ values, or the observed correlation could just be a systematic deviation of the biomolecules from the corresponding equilibrium state ${ }^{[11]}$.

We noticed that the ${ }^{13} \beta-\delta^{13} \mathrm{C}$ correlation ${ }^{[4-6]}$ uses the average isotope composition of a given system as the reference for its ${ }^{13} \beta-\delta^{13} \mathrm{C}$ correlation. In a system consisting of multiple components, if we choose a component as a reference for mutual comparison, even if the reference is the average stable isotope composition of the compounds of interest, we have effectively assigned that reference to be at equilibrium. The use of such a reference is not mathematically rigorous and can often be misleading when dealing with a complex nonequilibrium system. This is simply because we do not know a priori which compound or set of compounds represents the state of isotope equilibrium. In this contribution, we propose that the deviation of a complex system from its equilibrium state can be rigorously described as a graph problem, as applied in discrete mathematics. It can be represented by an apparent $\alpha$ difference matrix $(|\Delta \alpha|)$, i.e. the difference matrix between the observed isotope difference matrix $\left(\left|\alpha_{\mathrm{ob}}\right|\right)$ and its corresponding equilibrium fractionation matrix $\left(\left|\alpha_{\mathrm{eq}}\right|\right)$. We show that the $|\Delta \alpha|$ matrix can be used to assess apparent local equilibrium in an overall non-equilibrium complex system, both in general and in specific cases.

\section{Methods for evaluating a system at disequilibrium states}

A system can have only one possible distribution, i.e. one set of $\alpha$ values, at isotope equilibrium but numerous possible distributions at isotope disequilibrium.

If we treat each biomolecule as a node with one variable (the $\delta^{\prime}$ or $1000 \ln \beta$ value) and the apparent difference in isotope composition as distance, the distribution of the isotope composition of all the biomolecules in question in a system is, mathematically, a graph problem. Distance matrix is an exhaustive tabulation that consists of pairwise relationships between components. It is an abstraction that represents the relative position information of a graph, which can be used to describe the distribution of a graph and the similarities between graphs ${ }^{[13]}$. We have applied here the concept of distance matrix to describe the distribution of isotope compositions of multiple components in a complex system.

For a system with $\mathrm{K}$ components, the isotope composition distribution can be described by a $\mathrm{K} \times \mathrm{K}$ matrix: 
$|\alpha|=\left|\begin{array}{ccccc}\alpha_{11} & \alpha_{12} & \alpha_{13} & \cdots & \alpha_{1 K} \\ \alpha_{21} & \alpha_{22} & \alpha_{23} & \cdots & \alpha_{2 K} \\ \vdots & \vdots & \vdots & \ddots & \vdots \\ \alpha_{K 1} & \alpha_{K 2} & \alpha_{K 3} & \cdots & \alpha_{K K}\end{array}\right|$

where $\alpha_{\mathrm{ij}}\left(\alpha_{\mathrm{ij}}=\delta{ }_{\mathrm{i}}-\delta^{\prime}{ }_{\mathrm{j}}\right)$ denotes the difference in isotope compositions between component $\mathrm{i}$ and component $\mathrm{j}$. For the predicted equilibrium state, $\alpha_{\mathrm{ij}}=1000 \ln \beta_{\mathrm{i}}-1000 \ln \beta_{\mathrm{j}}$.

The difference between the observed isotope difference $\left|\alpha_{\mathrm{ob}}\right|$ and the theoretical calculated equilibrium isotope difference $\left|\alpha_{\text {eq }}\right|$ :

$|\Delta \alpha|=\left|\alpha_{o b}\right|-\left|\alpha_{e q}\right|$

describes the deviation of the measured isotope composition distribution from the equilibrium state. $|\Delta \alpha|$ is a zero matrix when the system reaches equilibrium. Thus, the $|\Delta \alpha|$ method removes the influence of isotope source and considers directly the distribution pattern of isotope composition among components. Thus, different sets of isotope compositions, regardless of the background isotope composition, can be compared directly.

$|\Delta \alpha|$ matrices can be visualized with a heat map, in which the absolute values of apparent $\alpha$ difference (the deviation from equilibrium isotope fractionation) are illustrated in color. Our heat map illustrates only the lower triangle of the matrix without a diagonal because the matrix is symmetric with a diagonal of 0 (Figures 1 and 2).

In addition to the overall isotope distribution, each $\Delta \alpha_{i j}$ measures the degree of deviation of the apparent $\alpha$ difference between components $\mathrm{i}$ and $\mathrm{j}$ from their respective equilibrium values. Thus, the apparent local equilibrium can also be identified using $|\Delta \alpha|$ matrices by looking for components with $\left|\Delta \alpha_{\mathrm{ij}}\right| \approx 0$. The heat map visually helps inspect the closeness of any paired components to their isotope equilibrium state. In the next section, we re-process some of the published data using the $|\Delta \alpha|$ method to examine if there is indeed local equilibrium among amino acids (AAs) in living systems.

\section{Applications}

Using equilibrium $\beta$ values of AAs calculated by Rustad ${ }^{[14]}$, we evaluate two cases, potato and a green alga, in the studies of thermodynamic order ${ }^{[4]}$. AAs are grouped into different families on the basis of their properties and biosynthetic pathways. The six families are: (i) the $\alpha-$ ketoglutarate family, including glutamic acid, glutamine, proline and arginine; (ii) the 3phosphoglycerate family, including serine, glycine and cysteine; (iii) the oxaloacetate family, including aspartic acid, asparagine, methionine, threonine, lysine and isoleucine; (iv) the pyruvate family, including alanine, valine and leucine; (v) the phosphoenol pyruvate and erythrose 4-phosphate family, including tryptophan, phenylalanine and tyrosine; and (vi) the ribose 5-phosphate family, histidine. 


\subsection{Potato leaves and tubers}

The very existence of "thermodynamic order" in AAs in potato leaves ${ }^{[15]}$ has been the subject of considerable debate $^{[4,5,11,12]}$. Our constructed heat maps of $|\Delta \alpha|$ matrices (Figure 1) show that local equilibrium does exist among the synthetic families of AAs in potato leaves.

Figure 1 shows that, in potato leaves, AAs in the same synthetic families are close to apparent carbon isotope equilibrium with each other: (i) alanine and valine are in the pyruvate family $\left(\Delta \alpha_{\text {ala-val }} 0.9 \%\right.$ ); (ii) lysine is in the oxaloacetate family, synthesized from aspartic acid $\left(\Delta \alpha_{\text {asp-lys }}\right.$ $0.4 \%$ ); (iii) proline is in the $\alpha$-ketoglutarate family, synthesized from glutamic acid ( $\Delta \alpha_{\text {glu-pro }}$ $0.4 \%$ ); and (iv) phenylalanine and tyrosine are both synthesized from phosphoenol pyruvate and erythrose-4-phosphate $\left(\Delta \alpha_{\text {phe-tyr }} 0.1 \%\right.$ ). An apparent close-to-equilibrium relationship also exists among proline, lysine, glutamic acid, and aspartic acid (oxaloacetate family and $\alpha$-ketoglutarate family), which may be caused by isotope exchange between their precursors, $\alpha$-ketoglutarate and oxaloacetate, in the citric acid cycle ${ }^{[16]}$. In addition, oxaloacetate can be synthesized from pyruvate, and the metabolic product of valine, succinyl-CoA, can also enter the citric acid cycle, which may explain the apparently close-to-equilibrium relationship between valine and aspartic acid $\left(\Delta \alpha_{\text {asp-val }} 0.7 \%\right.$ o).

Interestingly, compared with potato leaves, the carbon isotope composition of AAs in the tubers of the same potato displays a different distribution pattern. Specifically, the apparent close-toequilibrium isotope differences among synthetic families that are observed in leaves are absent from the tubers. However, other apparent close-to-equilibrium isotope differences appear, e.g. $\Delta \alpha_{\text {lys-val }} 0.5 \%, \Delta \alpha_{\text {glu-val }} 0.5 \%, \Delta \alpha_{\text {glu-asp }} 0.2 \%, \Delta \alpha_{\text {pro-tyr }} 0.2 \%$, and $\Delta \alpha_{\text {phe-ser }} 0.0 \%$. The difference in $|\Delta \alpha|$ matrices for potato leaves and tubers indicates that different degrees of reversibility in AAs synthetic pathways or entirely different synthetic pathways may exist in the two different potato parts. Conventionally, AAs synthesis is believed to take place entirely in leaves ${ }^{[17]}$. If AAs in tubers are synthesized in leaves and distributed to the tuber from leaves alone, the $|\Delta \alpha|$ for tubers should be the same as for leaves. Recent studies reveal, however, that some AAs, e.g. asparagine, can be synthesized in situ in potato tubers ${ }^{[18]}$. Our results support the suggestion by Muttucumaru et al that AAs synthesis may not occur entirely in leaves. In addition, the degradation of AAs in tubers can alter the isotope distribution, which may lead to the disappearance of the apparent local equilibrium that was initially transferred from potato leaves.

\subsection{The green alga Chlorella pyrenoidosa}

In addition to the apparently well-correlated ${ }^{13} \beta-\delta^{13} \mathrm{C}$ cases, some cases of poor ${ }^{13} \beta-\delta^{13} \mathrm{C}$ correlations have been reported ${ }^{[4]}$. The poor correlation was interpreted as an evidence for less expressed reversibility in enzymatic reactions. For instance, a "thermodynamic order" was shown to exist among different AAs in the autotrophic green alga, Chlorella pyrenoidosa, but it was absent from a glucose-cultured group. This difference was attributed to the disruption of "thermodynamic order" when the alga was fed with external nutrients or another carbon source 
such as glucose. We have reanalyzed the data from Abelson and Hoering ${ }^{[19]}$ ) and the results are presented in Figure 2.

The study of ${ }^{13} \beta-\delta^{13} \mathrm{C}$ correlations led to a conclusion that the degree of reversibility of enzymatic reactions in the glucose-cultured green alga was much poorer than in the autotrophic one $^{[4]}$. However, our $|\Delta \alpha|$ matrix analysis shows that a different apparent local equilibrium exists among different AAs regardless of the culture method or nutrition source of the alga. Opposite to the prediction that an external carbon source disturbs thermodynamic order in organisms ${ }^{4]}$, the pyruvate family (alanine and leucine, $\Delta \alpha_{\text {ala-leu }} 0.3 \%$ ) and oxaloacetate family (isoleucine and lysine, $\Delta \alpha_{\text {ile-lys }} 0.1 \%$ ) are closer to apparent equilibrium in the glucose-cultured alga than in the autotrophic alga $\left(\Delta \alpha_{\text {ala-leu }} 7.1 \%\right.$ o, $\Delta \alpha_{\text {ile-lys }} 1.8 \%$ o). It would be expected that the isotope distribution would differ in the two differently cultured algae, since the external carbon source would alter the metabolic kinetics. The current data cannot lead to the conclusion that one of the systems is more in equilibrium than the other.

\section{Implications and Future Works}

Our $|\Delta \alpha|$ matrix approach for analyzing intermolecular isotope distribution of a complex system can also be applied to those of intramolecular systems. The analytical results can serve as forensic identifiers for biosynthetic pathways and their reversibility, metabolic states, environmental conditions, and other information. For instance, different mechanism of fatty acids production in $E$. coli and $S$. cerevisiae are deciphered using intramolecular $\delta^{13} \mathrm{C}$ variations ${ }^{[20,21]}$. Before we understand intramolecular $\delta^{13} \mathrm{C}$ variations, we need to find an appropriate way to describe this varitation. Our $|\Delta \alpha|$ matrix serves to this purpose by looking at a system as a whole. Another important condition in applying the $|\Delta \alpha|$ matrix approach is to know the equilibrium state, from which the disequilibrium state can be evaluated. Some average and site-specific ${ }^{13} \beta$ values for organic molecules have been calculated ${ }^{[4,14,22,23]}$. The ab initio calculation can be used to estimate ${ }^{13} \beta$ values theoretically.

\section{Conclusions}

We have demonstrated that the $|\Delta \alpha|$ matrix describes rigorously the stable isotope distributions in a system that has many interacting components. The previous simple linear $\delta$ - $\beta$ correlation method is inadequate mainly because we are dealing with complex, multi-component systems in which each compound or site has its own predicted difference between measured and equilibrium values. The $|\Delta \alpha|$ approach can help identify apparent local equilibrium in an inherently disequilibrium system. The applications of the $|\Delta \alpha|$ matrix to the carbon isotope composition of different AAs from earlier published data, e.g. potato leaves and tubers, as well as the autotrophic vs glucose-cultured green alga, Chlorella pyrenoidosa, shows that a 
remarkable apparent local equilibrium does exist in potato leaves and also to a certain degree in both example of the green alga.

\section{Acknowledgments}

We thank J.A. Hayles, G.L. Waldrop, K.S. McCarter and Y. Zhou for constructive comments and discussion on this manuscript. We appreciate J.R. Maxwell for improving this manuscript. H.M. is grateful for funding support from the strategic priority research program (B) of CAS (XDB18010104) and Chinese NSF project (41490635).

\section{References}

1. Igamberdiev AU, Kleczkowski LA. Metabolic systems maintain stable non - equilibrium via thermodynamic buffering. BioEssays 2009;31(10):1091-1099. doi: 10.1002/bies.200900057.

2. Igamberdiev AU, Kleczkowski LA. Optimization of $\mathrm{CO}_{2}$ fixation in photosynthetic cells via thermodynamic buffering. BioSystems 2011;103(2):224-229. doi: 10.1016/j.biosystems.2010.10.001.

3. Morandini P. Rethinking metabolic control. Plant Sci 2009;176(4):441-451. doi: 10.1016/j.plantsci.2009.01.005.

4. Galimov EM. The Biological Fractionation of Isotopes. Orlando, FL: Academic Press; 1985.

5. Galimov EM. Phenomenon of life: between equilibrium and non-linearity. Orig Life Evol Biosph 2004;34(6):599-613. doi: 10.1023/B:ORIG.0000043131.86328.9d.

6. Galimov EM. Phenomenon of life: between equilibrium and non-linearity. Origin and principles of evolution. Geochem Int 2006;44(1):S1-S95. doi: 10.1134/S0016702906130015.

7. Hayes JM. Fractionation of carbon and hydrogen isotopes in biosynthetic processes. Rev. Mineral Geochem 2001;43(1):225-277. doi: 10.2138/gsrmg.43.1.225.

8. Urey HC. The thermodynamic properties of isotopic substances. J Chem Soc 1947;562-581.

9. Bigeleisen J, Mayer MG. Calculation of equilibrium constants for isotopic exchange reactions. J Chem Phys 1947;15(5):261-267.

10. Buchachenko AL. On isotope fractionation in enzymatic reaction. Russ J Phys Chem A 2007;81(5):836-836. doi: 10.1134/s0036024407050330.

11. Schmidt HL. Fundamentals and systematics of the non-statistical distributions of isotopes in natural compounds. Naturwissenschaften 2003;90(12):537-552. doi: 10.1007/s00114003-0485-5.

12. Schmidt HL, Robins RJ, Werner RA. Multi-factorial in vivo stable isotope fractionation: causes, correlations, consequences and applications. Isot Environ Health Stud 2015;51(1):155-199. doi: 10.1080/10256016.2015.1014355.

13. Dattorro J. Convex optimization \& Euclidean distance geometry Lulu. com; 2010.

This article is protected by copyright. All rights reserved. 
14. Rustad JR. Ab initio calculation of the carbon isotope signatures of amino acids. Org Geochem 2009;40(6):720-723. doi: 10.1016/j.orggeochem.2009.03.003.

15. Gleixner G, Scrimgeour C, Schmidt H-L, Viola R. Stable isotope distribution in the major metabolites of source and sink organs of Solanum tuberosum L.: a powerful tool in the study of metabolic partitioning in intact plants. Planta 1998;207(2):241-245. doi: 10.1007/s004250050479.

16. Bender DA. Amino acid metabolism. John Wiley \& Sons; 2012.

17. Foyer $\mathrm{CH}$, Parry M, Noctor G. Markers and signals associated with nitrogen assimilation in higher plants. J Exp Bot 2003;54(382):585-593.

18. Muttucumaru N, Keys AJ, Parry MA, Powers SJ, Halford NG. Photosynthetic assimilation of $14 \mathrm{C}$ into amino acids in potato (Solanumtuberosum) and asparagine in the tubers. Planta 2014;239(1):161-170.

19. Abelson PH, Hoering TC. Carbon isotope fractionation in formation of amino acids by photosynthetic organisms. Proc Natl Acad Sci (USA) 1961;47(5):623-632.

20. Monson KD, Hayes J, Biosynthetic control of the natural abundance of Carbon 13 at specific positions within fatty acids in Escherichia coli. Evidence regarding the coupling of fatty acid and phospholipid synthesis. J Biol Chem 1980;255 (23):11435-11441.

21. Monson KD; Hayes J, Biosynthetic control of the natural abundance of carbon 13 at specific positions within fatty acids in Saccharomyces cerevisiae. Isotopic fractionation in lipid synthesis as evidence for peroxisomal regulation. J Biol Chem 1982;257 (10):5568-5575.

22. Webb, MA, Miller III, TF. Position-specific and clumped stable isotope studies: Comparison of the Urey and path-integral approaches for carbon dioxide, nitrous oxide, methane, and propane. J Phys Chem A 2014;118(2):467-474.

23. Piasecki, A, Sessions, A, Lawson, M, et al. Position-specific ${ }^{13} \mathrm{C}$ distributions within propane from experiments and natural gas samples. Geochim Cosmochim Acta 2017;220(1):110-124.

This article is protected by copyright. All rights reserved. 

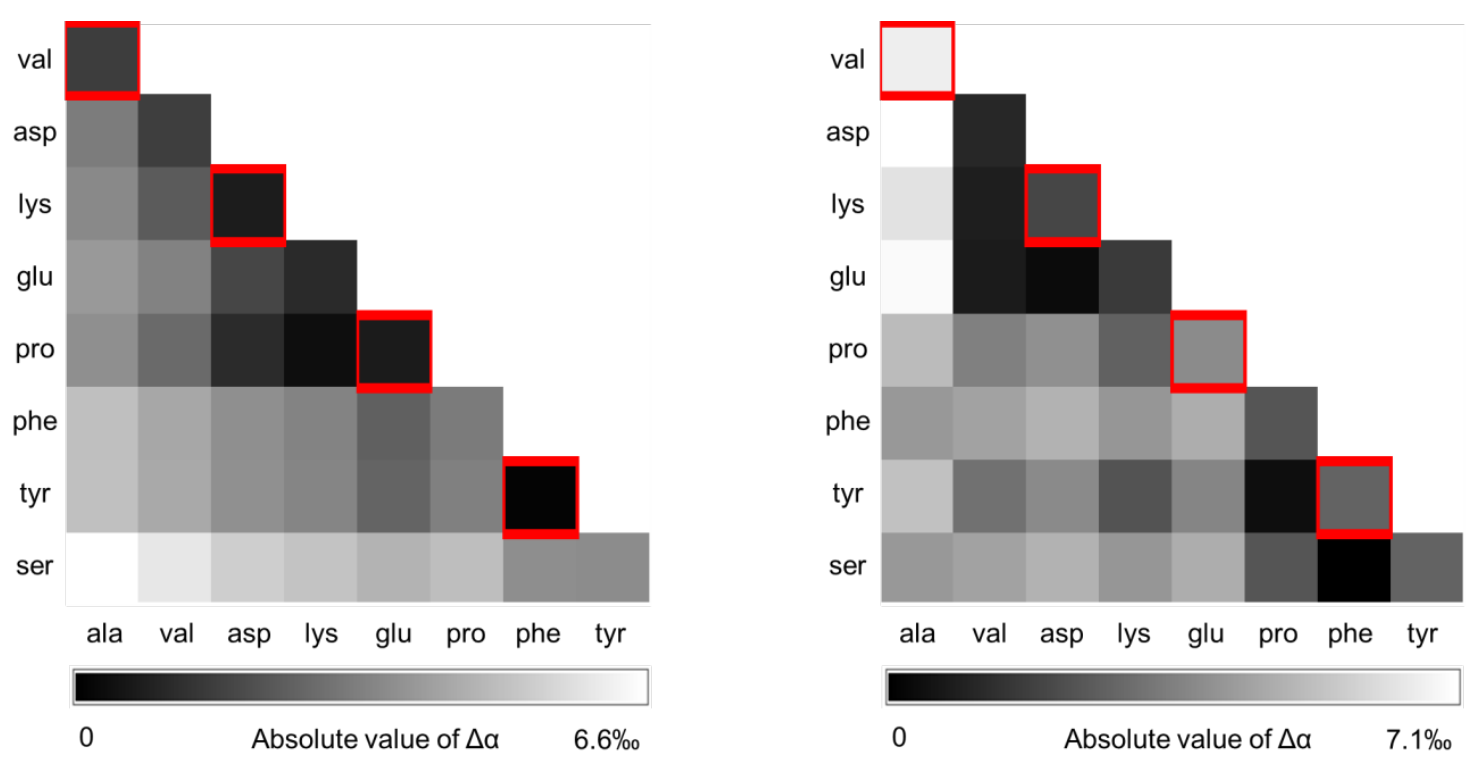

Fig. 1. $|\Delta \alpha|$ matrix heat maps for carbon isotope composition of AAs in potato leaves (left) and tubers (right). Each cell corresponds to the apparent $\alpha$ difference $(\Delta \alpha)$ for correspondingly paired AAs. Its value is represented in a color scale, with black for close to apparent equilibrium and white for the largest deviation. Red squares mark paired AAs of the same synthetic family, ala, alanine; val, valine; asp, aspartic acid; lys, lysine; glu, glutamic acid; pro, proline; phe, phenylalanine; tyr, tyrosine; ser, serine.
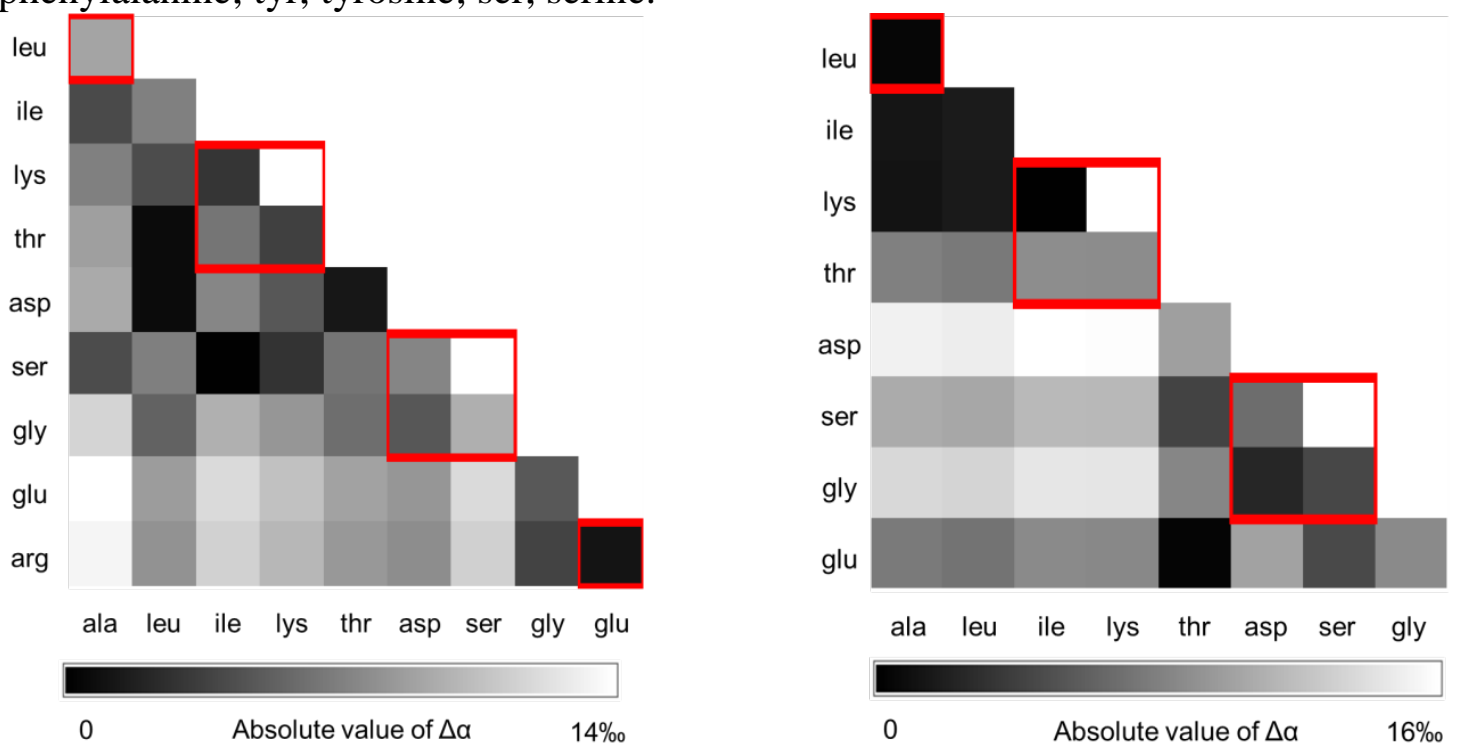

Fig. 2. $|\Delta \alpha|$ matrix heat maps for carbon isotope composition of AAs in autotrophic (left) and glucose-cultured (right) Chlorella pyrenoidosa. Each cell corresponds to the apparent $\alpha$ difference $(\Delta \alpha)$ for a pair of AAs. The value of $\Delta \alpha$ is represented on a color scale, with black for close to apparent equilibrium and white for the largest deviation. Red squares mark paired AAs of the same synthetic family; for abbreviations see Figure 1. 